Case report

\title{
Retreatment of symptomatic hepatitis $C$ virus genotype 3 associated mixed cryoglobulinemia with sofosbuvir and ribavirin: a case report
}

\author{
Dorota Zarębska-Michaluk, Piotr M. Stępień, Katarzyna Paluch, Paweł Pabjan \\ Clinic of Infectious Diseases, Regional Polyclinical Hospital, Kielce, Poland
}

\begin{abstract}
A 52-year-old woman with chronic hepatitis C virus genotype 3 infection developed clinically symptomatic mixed cryoglobulinemia. She started pegylated interferon and ribavirin therapy and in week 12 became negative for HCV RNA with resolution of clinical signs of cryoglobulinemia. The dual treatment was discontinued due to interferon-related bilateral retinopathy. After therapy cessation, relapse of HCV RNA and recurrence of symptomatic cryoglobulinemia were observed. While waiting for the antiviral retreatment option she developed glomerulonephritis with renal impairment. Successful HCV eradication was achieved with 24-week treatment of sofosbuvir and ribavirin despite this regimen being considered as suboptimal therapy for genotype $3 \mathrm{HCV}$ infection. A sustained virological response resulted in lasting resolution of clinical symptoms of mixed cryoglobulinemia.
\end{abstract}

Key words: cryoglobulinemia, genotype 3, sofosbuvir.

Address for correspondence

Dorota Zarębska-Michaluk, Clinic of Infectious Diseases, Regional Polyclinical Hospital, 7 Radiowa St., 25-317 Kielce, Poland, phone: +4841368 22 62, e-mail: dorota1010@tlen.pl

\section{Introduction}

Chronic infection with hepatitis $\mathrm{C}$ virus (HCV) is estimated to affect approximately $3 \%$ of the global population. Since the virus is not only hepatotropic, the health issues related to HCV infection are not limited to the liver. A large spectrum of extrahepatic manifestations (EM) associated with chronic hepatitis $\mathrm{C}(\mathrm{CHC})$ have been reported. They may result from the immunological pathway as well as from direct action of the virus and replication in the affected tissues beyond the liver.

On the top of the list of extrahepatic manifestations is mixed cryoglobulinemia (MC), the most common and most documented HCV-related condition. MC is defined as an immune-mediated disorder caused by cryoglobulins - immunoglobulins which undergo reversible precipitation in serum at a temperature below $37^{\circ} \mathrm{C}$ [1]. The majority of patients with MC have only altered laboratory data (presence of cryoglobulins, positive test for rheumatoid factor, hypocomplementemia), but some of them present symptoms of varying intensity. Clinical signs of MC result from small-to-medium sized vessel vasculitis, and the classic triad of MC comprises purpura, asthenia, and arthralgia. In some patients more serious symptoms are present in the form of glomerulonephritis, peripheral neuropathy or severe systemic vasculitis. Because of the crucial role of HCV infection in the mechanism of MC, antiviral treatment was proposed as a first line therapy to treat this condition [2]. Until recently, $\mathrm{CHC}$ patients with mild or moderate MC-related vasculitis were treated with pegylated interferon (PEG-IFN) and ribavirin (RBV). In severe cases or in patients ineligible for IFN, symptomatic therapy with the anti-CD20 monoclonal antibody rituximab, corticosteroids, and plasmapheresis was considered [3]. In the era of direct acting antivirals (DAA) there are limited data available on patients with HCV-related mixed cryoglobulinemia on antiviral treatment [4-11]. 


\section{Case description}

In July 2010, a 52-year-old woman was admitted to the Dermatology Department complaining of palpable purpura in both lower legs and arthralgia in her knees. Leukocytoclastic vasculitis was diagnosed based on skin biopsy. In a routine laboratory test HCV antibodies were detected. Then she was referred to the Infectious Diseases Department for further evaluation of hepatitis $\mathrm{C}$ infection. Her medical history was not significant besides blood transfusion at age 24 due to anemia after delivery. Serum levels of aminotransferases were slightly elevated, HCV RNA was positive, genotype (GT) 3. Liver biopsy revealed chronic hepatitis with moderate fibrosis (stage 3 in Ishak score) and steatohepatitis (grade 2 on Brunt scale). Anti-HBc-total and antinuclear antibodies were negative. Mixed cryoglobulinemia type II, with the presence of circulating cryoglobulins consisting of polyclonal IgG and monoclonal IgM (with activity of rheumatoid factor), was diagnosed. Antiviral treatment of PEG-IFN $\alpha-2 b$ and RBV was initiated in November 2010. In the course of therapy we observed reduction and then resolution of purpura and arthralgia. Baseline HCV RNA was $1.2 \times 10^{6} \mathrm{IU} / \mathrm{ml}$ and became negative at week 12 as well as the cryoglobulin test. At week 20 treatment was discontinued because of a serious adverse event - bilateral interferon-associated retinopathy with visual impairment; at the time of discontinuation HCV RNA was negative. Then she was treated by an ophthalmologist with a high dose of steroids (four weeks) and pentoxifylline. After 8 weeks of antiviral treatment discontinuation, relapse of $\mathrm{HCV}$ RNA and recurrence of symptomatic cryoglobulinemia were observed. She was monitored by an ophthalmologist and a hepatologist for the next few years. In that period gradual improvement, but not full recovery, of the visual acuity was observed. Purpura of the lower legs was recurrent with higher intensity in summer months. In March 2014 she developed glomerulonephritis with peripheral edema and arterial hypertension. Urinalysis showed moderate proteinuria (up to $1.6 \mathrm{~g} / 24 \mathrm{~h}$ ) and hematuria with sediment red blood cells more than 100 per high power field (HPF). Glomerular filtration rate (GFR) was $49 \mathrm{ml} / \mathrm{min} / 1.73 \mathrm{~m}^{2}$ and kidney dysfunction stage 3 was diagnosed. Renal biopsy was not performed, but since she was HCV positive and had cryoglobulinemia, the diagnosis of HCV-related cryoglobulinemic glomerulopathy was determined by a nephrologist. In the next months arterial pressure normalized on antihypertensive drugs and proteinuria spontaneously decreased to a value below $0.5 \mathrm{~g} / 24$ hours. GFR remained in the range $40-59 \mathrm{ml} / \mathrm{min} / 1.73 \mathrm{~m}^{2}$. The patient was waiting for DAA drugs with activity against genotype 3
HCV. Finally, in December 2015 she started sofosbuvir (SOF) $400 \mathrm{mg}$ and RBV $800 \mathrm{mg}$ daily. At baseline she had clinical symptoms of mixed cryoglobulinemia in the form of lower leg purpura, and presence of cryoglobulins. Liver stiffness measured by shear-wave elastography was $7.6 \mathrm{Kpa}$, and laboratory parameters were as follows: HCV RNA $1.33 \times 10^{7} \mathrm{IU} / \mathrm{ml}$, total bilirubin $0.8 \mathrm{mg} / \mathrm{dl}$, albumin $4.2 \mathrm{~g} / \mathrm{dl}$, normal aminotransferases: aspartate (AST) $39 \mathrm{IU} / 1$ and alanine (ALT) $34 \mathrm{IU} / \mathrm{l}$, creatinine $1.32 \mathrm{mg} / \mathrm{dl}$, GFR $44 \mathrm{ml} / \mathrm{min} / 1.73 \mathrm{~m}^{2}$, glucose $98 \mathrm{mg} / \mathrm{dl}$, hemoglobin $12.1 \mathrm{~g} / \mathrm{dl}$, white blood cells $3550 / \mu \mathrm{l}$, platelets $185 \times 10^{5} / \mu \mathrm{l}$, erythrocyturia $10-20$ cells/ HPF with proteinuria $1.48 \mathrm{~g} / 24$ hours. She took amlodipine, torasemide and spironolactone; no drug interactions with sofosbuvir were expected so no dose modifications were made. By week $4 \mathrm{HCV}$ RNA was negative, purpura of the lower legs disappeared but the patient complained of weakness and the hemoglobin level decreased to $8.3 \mathrm{~g} / \mathrm{dl}$. Therefore, the ribavirin dose was reduced to $400 \mathrm{mg}$ until week 12. At that point of therapy the hemoglobin level increased to $11.5 \mathrm{~g} / \mathrm{dl}$ and the RBV dose returned to baseline. She completed 24 weeks of treatment with no other significant adverse events, kidney function was stable and cryoglobulins were negative at the end of treatment. Then she was followed up every 3 months. The patient achieved a sustained virological response - HCV RNA was negative at weeks 12 and 24 after the end of SOF+RBV therapy. At the most recent follow-up visit, 18 months after antiviral treatment completion, she remains clinically well, with no recurrence of mixed cryoglobulinemia symptoms. She is still aviremic, cryoglobulins in her blood are negative, kidney function is normal and no urinary abnormality is detected.

\section{Discussion}

Mixed cryoglobulinemia is the most frequent and best known HCV-related extrahepatic manifestation. In the majority of cases $\mathrm{MC}$ follows an asymptomatic course, but in some patients organ involvement due to systemic vasculitis may occur, as in the described patient. The most challenging symptomatic cases are those with kidney impairment. Since HCV plays a significant role in the pathomechanism of MC, antiviral therapy is recommended as a first line action [12]. Historically, many publications have confirmed resolution of clinical symptoms of MC in patients responsive to PEG-IFN and RBV or triple first-wave DAA-based therapy [13-17]. On the other hand, IFN could exacerbate or worsen organ symptoms related to $\mathrm{MC}$, and its use was limited because of numerous adverse events and lower efficacy compared to non-cryoglobuline- 
mic patients $[18,19]$. Additionally, some publications reported relapse of MC vasculitis despite achieving a sustained virological response after IFN-containing treatment $[20,21]$.

Introduction of interferon-free options with combination of DAA improved treatment safety and efficacy, but therapy of patients infected with GT3 did not demonstrate such a high response rate as GT1 patients. Currently available IFN-free therapeutic regimens for GT3 infected patients are based on sofosbuvir, which can be combined with RBV, daclatasvir (DCV) or velpatasvir (VEL). Data from phase III clinical trials reported a $84-85 \%$ sustained virological response rate for 24 -week SOF+RBV treatment, $89 \%$ for a 12 -week SOF+DCV regimen and $95 \%$ for a 12-week SOF+VEL schedule, whereas for GT1 patients IFN-free options provided efficacy up to $100 \%$ [22-26].

After a few years of access to IFN-free regimens, data concerning resolution of HCV-related mixed cryoglobulinemia symptoms following viral clearance as a result of this type of treatment are still limited, especially for GT3 patients. By describing this case we would like to provide additional evidence to support this issue.

Our patient demonstrated clinical symptoms of HCV-related MC in the form of purpura, arthralgia and glomerulonephritis with proteinuria and kidney impairment. She was treated with the SOF+RBV regimen, considered as a suboptimal option since the combination of SOF+DCV was not available because of non-reimbursement, and the SOF/VEL option had not been registered yet. She completed the treatment course as scheduled and the only adverse event observed during antiviral therapy was anemia related to ribavirin. It required RBV dose reduction with no impact on treatment efficacy. During therapy clinical symptoms of $\mathrm{MC}$ resolved, cryoglobulins became negative and renal function normalized simultaneously with HCV RNA eradication. Finally, she achieved a sustained virological response and 18 months after treatment completion she remains clinically well with no recurrence of MC signs.

Our findings confirmed recently published reports documenting the high efficacy with minor adverse effects of sofosbuvir-based regimens in populations of patients with HCV-related MC, but the small number of GT3 patients in reported cohorts reduces the power of evidence $[6-9,11]$. Only one case report of a persistent virological and clinical response as a result of a sofosbuvir-based regimen was published in a cryoglobulinemic GT3 patient with decompensated liver cirrhosis, but the treatment regimen also contained ledipasvir [27].

Despite recent advances in the therapy of hepatitis $\mathrm{C}$, the treatment in presence of symptomatic cryo- globulinemic vasculitis and glomerulonephritis with kidney impairment still remains a challenge. Our patient's history presents a best scenario; however, the results are obtained in a single case. From the learning point of view, further studies based on significant numbers of GT3 patients with HCV-related MC are needed to confirm the safety and long-term clinical response in this difficult-to-treat population.

\section{Disclosure}

Authors report no conflict of interest.

\section{References}

1. Lunel F, Musset L, Cacoub P, et al. Cryoglobulinemia in chronic liver diseases: role of hepatitis $C$ virus and liver damage. Gastroenterology 1994; 106: 1291-1300.

2. Pietrogrande M, De Vita S, Zignego AL, et al. Recommendations for the management of mixed cryoglobulinemia syndrome in hepatitis C virus-infected patients. Autoimmun Rev 2011; 10: 444-454.

3. Ferri C, Cacoub P, Mazzaro C, et al. Treatment with rituximab in patients with mixed cryoglobulinemia syndrome: Results of multicenter cohort study and review of the literature. Autoimmun Rev 2011; 11: 48-55.

4. Polo MN, Laufer N. Extrahepatic manifestations of HCV: the role of direct acting antivirals. Expert Rev Anti Infect Ther 2017; 15: 737-746.

5. Makara M, Sulyok M, Csacsovszki O, et al. Successful treatment of HCV-associated cryoglobulinemia with ombitasvir/paritaprevir/ritonavir, dasabuvir and ribavirin: A case report. J Clin Virol 2015; 72: 66-68.

6. Saadoun D, Thibault V, Si Ahmed SN, et al. Sofosbuvir plus ribavirin for hepatitis $\mathrm{C}$ virus-associated cryoglobulinaemia vasculitis: VASCUVALDIC study. Ann Rheum Dis 2015; 75: 1777-1782.

7. Sise ME, Bloom AK, Wisocky J, et al. Treatment of Hepatitis C Virus-Associated Mixed Cryoglobulinemia with Direct-Acting Antiviral Agents. Hepatology 2016; 63: 408-417.

8. Gragnani L, Viscentini M, Fognani E, et al. Prospective Study of Guideline-Tailored Therapy With Direct-Acting Antivirals for Heatitis C Virus-Associated Mixed Cryoglobulinemia. Hepatology 2016; 64: 1473-1482.

9. Gragnani L, Piluso A, Urraro T, et al. Virological and Clinical Response to Interferon-Free Regimens in Patients with HCV-Related Mixed Cryoglobulinemia: Preliminary Results of a Prospective Pilot Study. Curr Drug Targets 2017; 18: 772-785.

10. Mahieu R, Lavigne C, Urbanski G, et al. Successful Treatment Using Simeprevir, Sofosbuvir and Rituximab of a Severe Form of Hepatitis C Virus-related Mixed Cryoglobulinemia with Cardiac Involvement. Eur J Case Rep Intern Med 2015; https://doi. org/https://doi.org/10.12890/2015_000299.

11. Lauletta G, Russi S, Pavone F, et al. Direct-acting antiviral agents in the therapy of hepatitis $C$ virus-related mixed cryoglobulinemia: a single-centre experience. Arthritis Res Ther 2017; 19: 74.

12. Giuggioli D, Sebastiani M, Colaci M, et al. Treatment of HCVrelated mixed cryoglobulinemia. Curr Drug Targets 2017; 18: 794-802.

13. Gragnani L, Fognani E, Piluso A, et al. Long-Term Effect of HCV Eradication in patients With Mixed Cryoglobulinemia: 
A Prospective, Controlled, Open-Label, Cohort Study. Hepatology 2015; 61: 1145-1163.

14. Saadoun D, Resche-Rigon M, Thibault V, et al. Antiviral Therapy for Hepatitis C Virus-Associated Mixed Cryoglobulinemia Vasculitis. Arthritis Rheum 2006; 54: 3696-3706.

15. Fabrizi F, Dixit V, Messa P. Antiviral therapy of symptomatic HCV-associated mixed cryoglobulinemia: Meta-analysis of clinical studies. J Med Virol 2013; 85: 1019-1027.

16. Saadoun D, Resche-Rigon M, Pol S, et al. PegIFNa/ribavirin/ protease inhibitor combination in severe hepatitis $C$ virus-associated mixed cryoglobulinemia vasculitis. J Hepatol 2015; 62: 24-30.

17. Gragnani L, Fognani E, Piluso A, et al. Long-Term Effect of HCV Eradication in patients With Mixed Cryoglobulinemia: A Prospective, Controlled, Open-Label, Cohort Study. Hepatology 2015; 61: 1145-1163.

18. Batisse D, Karmochkine M, Jacquot C, et al. Sustained exacerbation of cryoglobulinemia-related vasculitis following treatment of hepatitis $\mathrm{C}$ with peginterferon alfa. Eur J Gastroenterol Hepatol 2004; 16: 701-703.

19. Gragnani L, Fabbrizzi A, Triboli E, et al. Triple antiviral therapy in hepatitis $\mathrm{C}$ virus infection with or without cryoglobulinemia: a prospective, controlled pilot study. Dig Liver Dis 2014; 46: 833-837.

20. Landau DA, Saadoun D, Halfon P, et al. Relapse of hepatitis C virus-associated mixed cryoglobulinemia vasculitis in patients with sustained viral response. Arthritis Rheum 2008; 58: 604-611.

21. Cornella SL, Stine JG, Kelly V, et al. Persistence of mixed cryoglobulinemia despite cure of hepatitis $\mathrm{C}$ with new oral antiviral therapy including direct-acting antiviral sofosbuvir: A case series. Postgrad Med 2015; 127: 413-417.

22. Zeuzem S, Dusheiko GM, Salupere R, et al. Sofosbuvir and ribavirin in HCV genotypes 2 and 3. Gastroenterology 2014; 370: 1993-2001.

23. Foster GR, Pianko S, Brown A, et al. Efficacy of sofosbuvir plus ribavirin with or without peginterferon-alfa in patients with hepatitis $\mathrm{C}$ virus genotype 3 infection and treatment-experienced patients with cirrhosis and hepatitis $\mathrm{C}$ virus genotype 2 infection. Gastroenterology 2015; 149: 1462-1470.

24. Nelson DR, Cooper JN, Lalezari JP, et al. Alloral 12-week treatment with daclatasvir plus sofosbuvir in patients with hepatitis C virus genotype 3 infection: ALLY-3 phase 3 study. Hepatology 2015; 61: 1127-1135.

25. Foster GR, Afdhal N, Roberts SK, et al. Sofosbuvir and Velpatasvir for HCV Genotype 2 and 3 Infection. N Engl J Med 2015; 373: 2608-2617.

26. D’Ambrosio R, Degasperi E, Colombo M, et al. Direct-acting antivirals: the endgame for hepatitis C? Curr Opin Virol 2017; 24: 31-37.

27. Flemming JA, Lowe CE. Successful treatment of hepatitis C, genotype 3, with sofosbuvir/ledipasvir in decompensated cirrhosis complicated by mixed cryoglobulinaemia. BMJ Case Rep 2016; doi: 10.1136/bcr-2016-215293. 
